\title{
Thermal diffusion measurements and simulations of binary mixtures of spherical molecules
}

\author{
Pavel Polyakov ${ }^{\text {a) }}$ \\ Forschungszentrum Jülich GmbH, IFF - Weiche Materie, D-52428 Jülich, Germany \\ Meimei Zhang ${ }^{\text {b) }}$ and Florian Müller-Plathe ${ }^{\text {c) }}$ \\ Eduard-Zintl-Institut für Anorganische und Physikalische Chemie, Technische Universität Darmstadt, \\ Petersenstrasse 20 D-64287 Darmstadt, Germany \\ Simone Wiegand ${ }^{\text {d) }}$ \\ Forschungszentrum Jülich GmbH, IFF - Weiche Materie, D-52428 Jülich, Germany
}

(Received 10 April 2007; accepted 10 May 2007; published online 2 July 2007)

\begin{abstract}
Thermal diffusion forced Rayleigh scattering measurements on binary mixtures of carbon tetrabromide $\left(\mathrm{CBr}_{4}\right)$, tetraethylsilane, and di-tert-butylsilane in carbon tetrachloride $\left(\mathrm{CCl}_{4}\right)$ are reported at different temperatures and concentrations. The Soret coefficient of $\mathrm{CBr}_{4}$ in $\mathrm{CCl}_{4}$ is positive and $S_{T}$ of both silanes in $\mathrm{CCl}_{4}$ is negative, which implies that the heavier component always moves to the cold side. This is the expected behavior for unpolar simple molecules. Both silanes have the same mass so the influence of the difference in shape and moment of inertia could be studied. For all three systems, $S_{T}$ decreases with decreasing $\mathrm{CCl}_{4}$ concentration. The results are discussed in the framework of thermodynamic theories and the Hildebrand parameter concept. Additionally, the Soret coefficients for both silane $/ \mathrm{CCl}_{4}$ systems were determined by nonequilibrium molecular-dynamics calculations. The simulations predict the correct direction of the thermophoretic motion and reflect the stronger drive toward the warm side for di-tert-butylsilane compared to the more symmetric tetraethylsilane. The values deviate systematically between $9 \%$ and 18\% from the experimental values. (C) 2007 American Institute of Physics.
\end{abstract}

[DOI: $10.1063 / 1.2746327]$

\section{INTRODUCTION}

Thermal diffusion describes the migration of molecules in a temperature gradient. The molecular origin of the effect, also called the Ludwig-Soret effect, is one of the unsolved problems in physical chemistry. In some cases, even qualitative predictions, which are of practical importance, are impossible. The main practical applications are separation processes $^{1,2}$ such as thermal field flow fractionation of polymers and colloids or isotope separation, characterization of geochemical processes, ${ }^{3,4}$ and combustion. ${ }^{5}$

Thermal diffusion in liquid mixtures of nonpolar fluids is known to reflect a range of microscopic properties such as the mass, size, and shape of the molecules as well as their interactions. ${ }^{6}$ In mixtures of polar liquids, specific interactions between the molecules dominate the thermal diffusion process, while mass and size of the molecules are most important in Lennard-Jones fluids. For liquids more complex than Lennard-Jones fluids, the Soret effect appears to depend on a delicate balance of the molecular properties of the components. $^{7-9}$

Even for fairly simple solvents without specific interactions, there is often no simple relation between the Soret

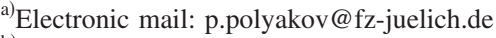

${ }^{b)}$ Electronic mail: m.zhang@theo.chemie.tu-darmstadt.de

${ }^{\text {c) }}$ Electronic mail: f.mueller-plathe@ theo.chemie.tu-darmstadt.de

d) Author to whom correspondence should be addressed. Electronic mail: s.wiegand@fz-juelich.de
}

coefficient and other physical properties. Especially if the solvent molecules are not approximately spherical, deviations from some simple rules of thumb are found: typically, the component with the larger mass or higher density moves to the cold side, and the effect becomes stronger if the components are less miscible. ${ }^{6}$ For isotopic mixtures of benzene and cyclohexane, it was found that the Soret coefficient can be written as a sum of three contributions: the mass difference, the difference in moment of inertia, and a chemical contribution. ${ }^{8}$ Recently, it was shown by equilibrium and nonequilibrium simulations of Lennard-Jones (LJ) mixtures that the composition dependence of the Soret effect is determined by the chemical contribution. ${ }^{10}$

The values of the Soret coefficient can also be compared with the cohesive energy density, also referred to as the Hildebrand solubility parameter. ${ }^{11-14}$ Two substances are mutually soluble if the free energy of mixing $\Delta G_{M}$ is negative. By definition, $\Delta G_{M}$ is given by

$$
\Delta G_{M}=\Delta H_{M}-T \Delta S_{M},
$$

where $\Delta H_{M}$ is the enthalpy of mixing per unit volume and $\Delta S_{M}$ is the entropy of mixing per unit volume. The value of $\Delta S_{M}$ is determined by the properties of the given mixture such as composition, compressibility, and specific interactions (e.g., hydrogen bonds). For mixtures of nearly spherical molecules without specific interactions and almost the same sizes, the value of $\Delta S_{M}$ is always positive, while there is a certain limiting positive value of $\Delta H_{M}$ above which dissolu- 
tion is impossible. Hildebrand first correlated solubility with the cohesive properties of the solvents and made the ansatz for a binary mixture

$$
\Delta H_{M}=\varphi_{1} \varphi_{2}\left(\delta_{1}^{2}-\delta_{2}^{2}\right)
$$

where $\varphi_{1}$ and $\varphi_{2}$ are volume fractions and $\delta_{1}$ and $\delta_{2}$ are the solubility parameters of the two components. Equation (2) predicts that $\Delta H_{M}=0$ if $\delta_{1}=\delta_{2}$. Hence, two substances with equal solubility parameters should be mutually soluble. In this limit, the two components become identical to each other, which makes the Soret coefficient of such mixture equal to zero. This hypothesis was confirmed by simulations of $S_{T}$ for binary LJ mixtures. ${ }^{12}$ It was found that the component with the larger Hildebrand parameter moves to the cold side. Later, this concept was successfully applied to non-LJ mixtures of components, which still can be mapped onto LJ spheres. $^{6}$

Thermodiffusion in Lennard-Jones binary fluids was investigated by molecular dynamic (MD) simulations. ${ }^{12,15}$ In those simulations, the influence of the different LJ parameters mass, atomic diameter, and interaction strength has been investigated systematically for binary mixtures. The ratio of one of the parameters was varied, while the others were fixed and equal for both compounds. The magnitude of the Soret coefficient was observed to depend on all three ratios. It was found that the heavier species, the smaller species, and species with higher interaction strengths tend to accumulate in the cold region.

The goal of this paper is the investigation of rather simple tetrahedral, nonpolar molecules, which can be well approximated by a spherical shape. Experiments on mixtures of tetrahedral liquids are in principle possible; the side atoms can be halogens, hydrogen, or alkyl groups $\left(\mathrm{CH}_{3}, \mathrm{C}_{2} \mathrm{H}_{5}\right)$, whereas the central atom can be turned into $\mathrm{C}, \mathrm{Si}, \mathrm{Ge}, \mathrm{Pb}$, $\mathrm{Sn}, \mathrm{Ti}$, or other transition metals. Unfortunately, many of the chemicals containing $\mathrm{Sn}$ and $\mathrm{Pb}$ are highly toxic. And, most of the others containing $\mathrm{Sn}, \mathrm{Ge}, \mathrm{Si}$, and $\mathrm{Ti}$ react with water vapor so that they are not suitable for systematic investigations with our present setup. After screening many spherical solvents, we picked carbon tetrachloride $\left(\mathrm{CCl}_{4}\right)$ (cf. Fig. 1), which has a rather small moment of inertia (cf. Table I). Additionally, we selected tetraethylsilane and di-tertbutylsilane, which differ in their moment of inertia, but have the same molar mass, which is slightly lower than the mass of $\mathrm{CCl}_{4}$ (cf. Table I). The size of both molecules corresponds roughly to a sphere with van der Waals radius of 3.51 and $3.57 \AA$, respectively (cf. Table I). Tetraethylsilane can better be approximated by a sphere than di-tert-butylsilane. As heavier compound we picked carbon tetrabromide $\left(\mathrm{CBr}_{4}\right)$, which is solid at room temperature, but dissolves in $\mathrm{CCl}_{4}$. Both carbon tetrahalides can be approximated by spheres with van der Waals radii of 2.91 and $2.73 \AA$, respectively (cf. Table I and Sec. II).

\section{EXPERIMENT}

\section{A. Sample preparation}

Carbon tetrachloride $(99.8 \%)$ was purchased from Fluka and carbon tetrabromide (99\%), tetraethylsilane (99\%) and

\section{$\mathrm{CBr}_{4}$}

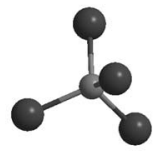

$\mathrm{Si}\left(\mathrm{C}_{2} \mathrm{H}_{5}\right)_{4}$

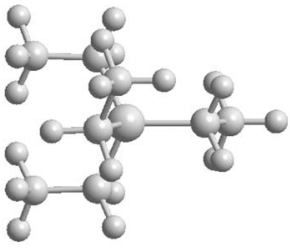

$\mathrm{SiH}_{2}\left[\left(\mathrm{CH}_{3}\right)_{3} \mathrm{C}\right]_{2}$
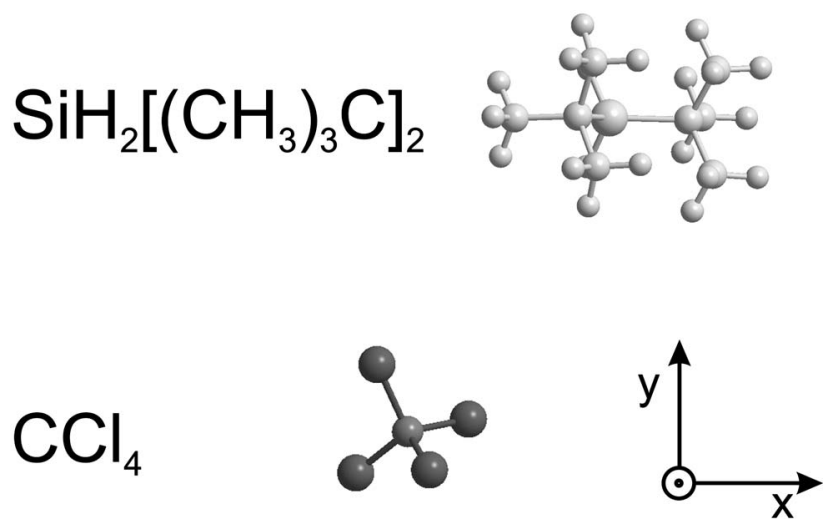

FIG. 1. Sketch of the investigated molecules carbon tetrabromide $\left(\mathrm{CBr}_{4}\right)$, tetraethylsilane, di-tert-butylsilane, and carbon tetrachloride $\left(\mathrm{CCl}_{4}\right)$ as balland-stick models. The diameter of the silanes is roughly twice as large as the diameter of $\mathrm{CBr}_{4}$ and $\mathrm{CCl}_{4}$. The reference system used for the calculation of the moments of inertia listed in Table $\mathrm{I}$ is indicated. The $z$ axis is perpendicular to the paper plane.

di-tert-butylsilane (97\%) were ordered from Aldrich. Carbon tetrabromide $\left(\mathrm{CBr}_{4}\right)$ forms white crystals at room temperature. The mole fraction was adjusted by the molecular mass and weight fraction of the components. At higher mole fractions $(x \gtrsim 0.5), \mathrm{CBr}_{4}$ becomes insoluble in $\mathrm{CCl}_{4}$. For the highest mole fraction investigated $(x=0.45), \mathrm{CBr}_{4}$ remains dissolved in $\mathrm{CCl}_{4}$ for at least one day. All samples contained a small amount of Quinizarin (Aldrich). The weight fraction of the dye is approximately $0.002 \mathrm{wt} \%$, corresponding to the optical density of 1.5 for a $1 \mathrm{~cm}$ cell. This amount ensures a sufficient temperature modulation of the optical grating. On the other hand, the quantity is small enough to avoid convection and contributions of the dye to the concentration signal. Approximately $2 \mathrm{ml}$ of the freshly prepared solution were filtered through $0.2 \mu \mathrm{m}$ filter (hydrophobic PTFE) into an optical quartz cell with $0.2 \mathrm{~mm}$ optical path length (Helma), which had been carefully cleaned of dust particles before usage. Experiments were performed in a temperature range from 20 to $40{ }^{\circ} \mathrm{C}$.

\section{B. Properties and physical parameters}

Table I lists the properties of the substances, such as molecular weight $M_{w}$, density $\rho$, van der Waals radius $r_{w}$, 
TABLE I. Physical properties of the investigated solvents $\left[M_{w}\right.$ : molecular weight, $\rho$ : density, $r_{w}$ : van der Waals radius, $T_{\text {boil }}$ : boiling temperature, $\delta$ : Hildebrand parameter at the boiling point, and $I_{i}$ : the moment-of-inertia components about the symmetry axes (cf. Fig. 1)]. The values of the main moments of inertia for all investigated compounds were calculated using an atomistic model for a single molecule in vacuum (Ref. 16).

\begin{tabular}{|c|c|c|c|c|c|c|c|c|}
\hline Solvent & $\begin{array}{l}M_{w}(\mathrm{~g} / \mathrm{mol}) \\
\text { Ref. } 17\end{array}$ & $\begin{array}{l}\rho\left(\mathrm{kg} / \mathrm{m}^{3}\right) \\
\text { Ref. } 17\end{array}$ & $r_{w}(\AA)$ & $\begin{array}{l}T_{\text {boil }}\left({ }^{\circ} \mathrm{C}\right) \\
\text { Ref. } 17 \\
\end{array}$ & $\delta\left(\mathrm{MPa}^{1 / 2}\right)$ & $\begin{array}{l}I_{x}\left(\mathrm{~g} \AA^{2} / \mathrm{mol}\right) \\
\text { Ref. } 16\end{array}$ & $\begin{array}{l}I_{y}\left(\mathrm{~g}^{2} / \mathrm{mol}\right) \\
\text { Ref. } 16\end{array}$ & $\begin{array}{l}I_{z}\left(\mathrm{~g}^{2} / \mathrm{mol}\right) \\
\text { Ref. } 16\end{array}$ \\
\hline $\mathrm{CBr}_{4}$ & 331.6 & solid & 2.91 & 190 & 18.1 & 796 & 796 & 796 \\
\hline $\mathrm{Si}\left(\mathrm{C}_{2} \mathrm{H}_{5}\right)_{4}$ & 144.3 & 0.761 & 3.51 & 153 & 13.4 & 452 & 492 & 593 \\
\hline$\left[\left(\mathrm{CH}_{3}\right)_{3} \mathrm{C}\right]_{2} \mathrm{SiH}_{2}$ & 144.3 & 0.729 & 3.57 & 129 & 12.7 & 246 & 637 & 650 \\
\hline $\mathrm{CCl}_{4}$ & 153.8 & 1.59 & 2.73 & 76 & 17.0 & 282 & 282 & 282 \\
\hline
\end{tabular}

boiling temperature $T_{\text {boil }}$, the Hildebrand parameter $\delta$ at the boiling point, and the moments of inertia about the symmetry axes $I_{i}$. We estimated $\delta$ by

$$
\delta=\left(\frac{\Delta H_{\text {vap }}-\mathrm{RT}}{V}\right)^{1 / 2}
$$

where $\Delta H_{\text {vap }}$ is the enthalpy of vaporization and $V$ is the molar volume of the pure component. The enthalpy of vaporization at the boiling point was estimated using Trouton's rule. Trouton's rule states that the entropy change for vaporization is approximately $88 \mathrm{~J} \mathrm{~mol}^{-1} \mathrm{~K}^{-1}$ and holds for many liquids to within $10 \%,{ }^{18}$

$$
\Delta H_{\text {vap }}=\Delta S_{\text {vap }} T_{\text {boil }} \text {. }
$$

The obtained values (cf. Table I) compare well with the literature values at lower temperatures $\left[13.9 \mathrm{MPa}^{1 / 2}\right.$ (Ref. 19) for tetraethylsilane and $17.3 \mathrm{MPa}^{1 / 2}$ (Ref. 20) for carbon tetrachloride, estimated from the enthalpy of vaporization at $298 \mathrm{~K}]$. For carbon tetrabromide we found only a value of $20.1 \mathrm{MPa}^{1 / 2},{ }^{19,21} 70 \mathrm{~K}$ below the boiling temperature. The fact that these three experimental values are slightly higher is consistent with the fact that the Hildebrand parameter increases with decreasing temperature.

Van der Waals radii for all substances were estimated using the van der Waals increment method ${ }^{22}$ (cf. Table I). This method gives the same value of $3.48 \AA$ for both tetraethylsilane and di-tert-butylsilane. Estimation from the liquid volume $^{20,22}$ for these two compounds gives 3.57 and $3.66 \AA$, respectively. The effective hard-sphere diameter of tetraethylsilane calculated from modified van der Waals equation of

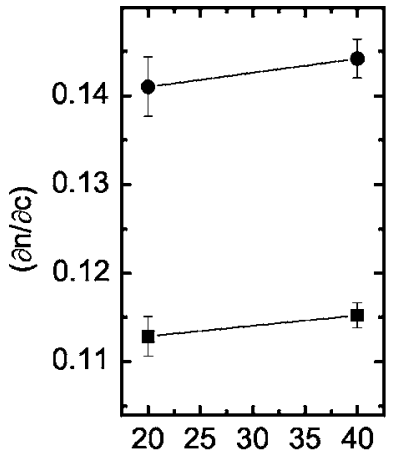

(a)

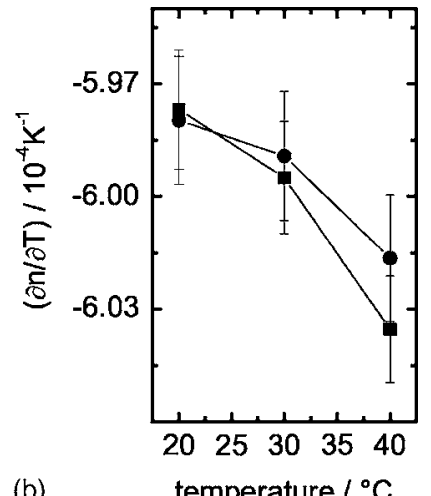

FIG. 2. Refractive index increments $(\partial n / \partial c)_{p, T}$ and $(\partial n / \partial T)_{p, c}$ of $\mathrm{CBr}_{4}$ in $\mathrm{CCl}_{4}$ as a function of temperature. The mole fractions of $\mathrm{CBr}_{4}$ are 0.1 ( and $0.25(\bullet)$ state is equal to $3.49 \AA{ }^{23}$ which agrees with our estimates. The averaged values of van der Waals radii for the silanes are listed in Table I.

\section{Refractive index increment measurements}

Refractive index increments with concentration $(\partial n / \partial c)_{p, T}$ at a constant pressure and temperature were measured using an Abbe refractometer. The temperature derivatives of the refractive index $(\partial n / \partial T)_{p, c}$ at a constant pressure and concentration were determined in temperature range $T \pm 3{ }^{\circ} \mathrm{C}$ using a Michelson interferometer. ${ }^{24}$

Figure 2 shows both refractive index increments at different temperatures and concentrations for $\mathrm{CBr}_{4}$ in $\mathrm{CCl}_{4}$; $(\partial n / \partial c)_{p, T}$ increases, while $(\partial n / \partial T)_{p, c}$ decreases with increasing temperature. The values for the higher concentration of $\mathrm{CBr}_{4}$ in $\mathrm{CCl}_{4}$ are slightly higher. The error bars, typically around $0.3 \%$, represent one standard deviation between repeated measurements. The refractive index increment with temperature is almost insensitive to the concentration. Figures 3 and 4 show refractive index increments at different temperatures and concentrations for $\mathrm{Si}\left(\mathrm{C}_{2} \mathrm{H}_{5}\right)_{4}$ and $\left[\left(\mathrm{CH}_{3}\right)_{3} \mathrm{C}\right]_{2} \mathrm{SiH}_{2}$ in $\mathrm{CCl}_{4}$.

\section{TDFRS experiment and data analysis}

The principle of the thermal diffusion forced Rayleigh scattering (TDFRS) method is described elsewhere in detail. ${ }^{25}$ An argon-ion laser $\left(\lambda_{w}=488 \mathrm{~nm}\right)$ is used for writing the grating. The laser beam is split into two writing beams of

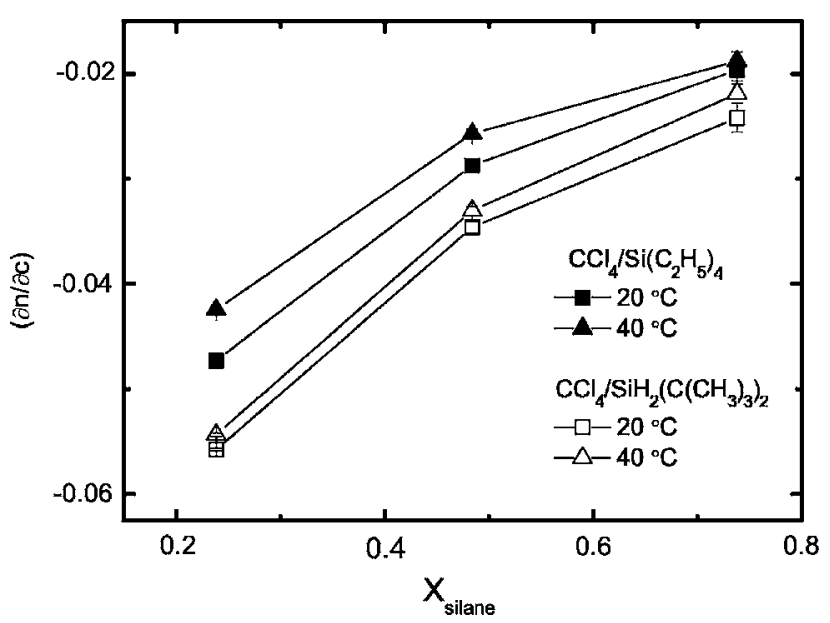

FIG. 3. Refractive index increments $(\partial n / \partial c)_{p, T}$ of tetraethylsilane (solid symbols) and di-tert-butylsilane (open symbols) in carbon tetrachloride as a function of concentration. 


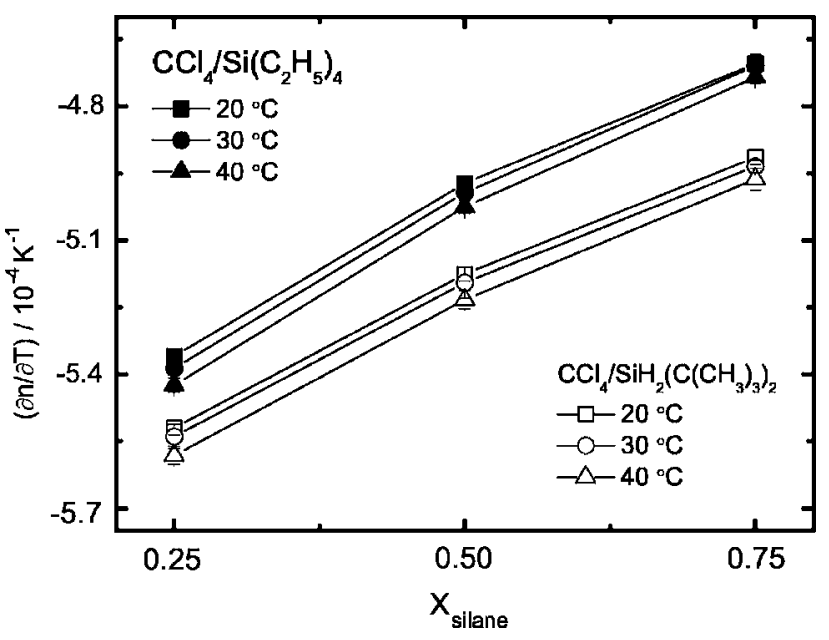

FIG. 4. Refractive index increments $(\partial n / \partial T)_{p, c}$ of tetraethylsilane (solid symbols) and di-tert-butylsilane (open symbols) in carbon tetrachloride as a function of concentration.

equal intensity by a beam splitter. An intensity grating is created in the sample by the interference of two laser beams. A small amount of dye in the sample converts the intensity grating into a temperature grating, which in turn causes a concentration grating by the effect of thermal diffusion. Both gratings contribute to a combined refractive index grating, which is read out by diffraction of a third laser beam $\left(\lambda_{r}\right.$ $=633 \mathrm{~nm}$ ). The heterodyne signal intensity of the read-out laser is proportional to the amplitude of the refractive index difference $\Delta n(T, c)$ as

$$
\Delta n(T, c)=\left(\frac{\partial n}{\partial T}\right)_{p, c} \Delta T+\left(\frac{\partial n}{\partial c}\right)_{p, T} \Delta c,
$$

where $\Delta T$ and $\Delta c$ are the difference in temperature and concentration, respectively.

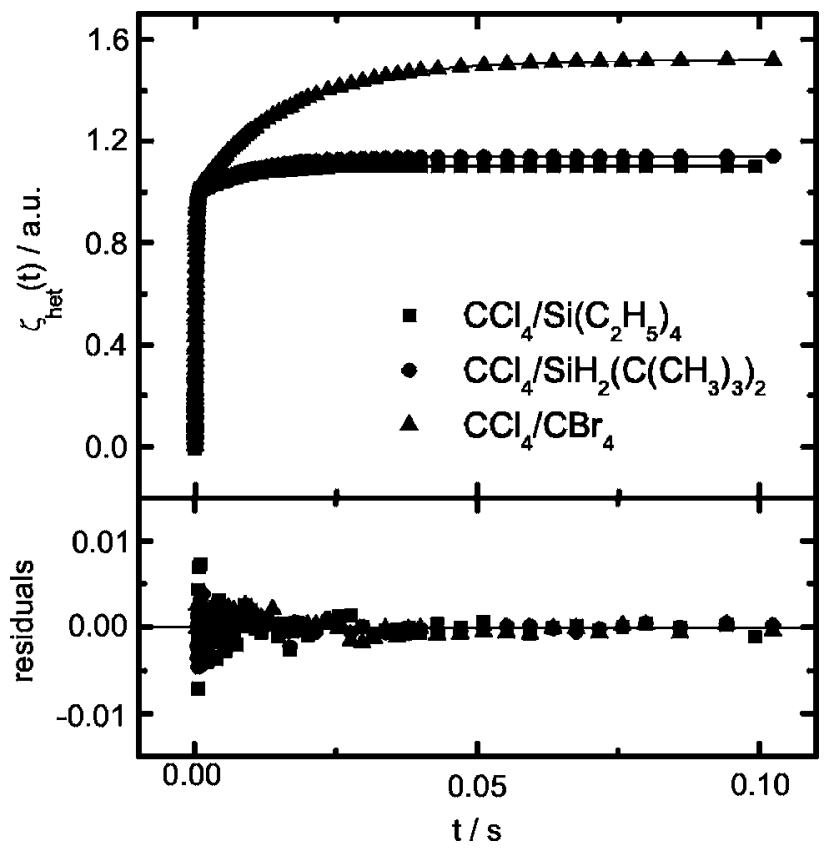

FIG. 5. Typical normalized TDFRS signals and the residuals plot to the fitting curve according to Eq. (7) of investigated mixtures at a temperature of $20{ }^{\circ} \mathrm{C}$. Mole fraction of $\mathrm{CCl}_{4}$ equals 0.75 . The solid lines refer to a fit according to Eq. (6).

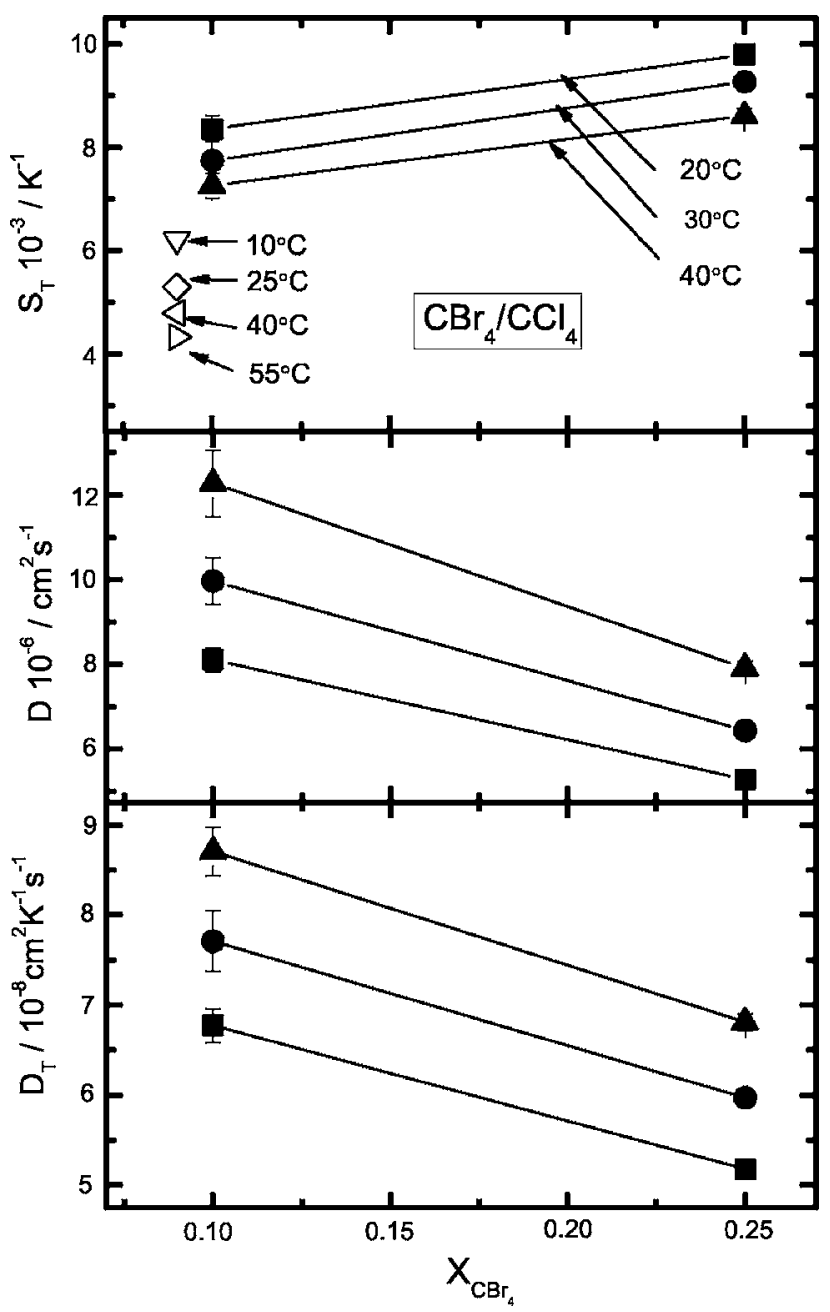

FIG. 6. Soret coefficient $S_{T}$, diffusion coefficient $D$, and thermal diffusion coefficient $D_{T}$ of $\mathrm{CBr}_{4}$ in $\mathrm{CCl}_{4}$ as a function of concentration. Solid symbols - our data; open symbols_-data from Saxton et al. (Ref. 26).

The total intensity $\zeta_{\text {het }}(t)$ normalized to the thermal signal is related to the Soret coefficient as

$$
\zeta_{\text {het }}(t)=1-\left(\frac{\partial n}{\partial T}\right)_{p, c}^{-1}\left(\frac{\partial n}{\partial c}\right)_{p, T} S_{\mathrm{T}} c(1-c)\left(1-e^{-q^{2} D t}\right),
$$

where $q$ is the grating vector and $D$ is the mutual diffusion coefficient.

To determine the transport coefficients, Eq. (6) is fitted to the measured heterodyne signal (Fig. 5) using contrast factors $(\partial n / \partial c)_{p, T}$ and $(\partial n / \partial T)_{p, c}$ which are measured separately. The residuals are small (less than 1\%) and show no systematic deviations (Fig. 5).

\section{RESULTS}

Figure 6 shows the concentration dependence of the transport coefficients for the mixture of $\mathrm{CBr}_{4}$ and $\mathrm{CCl}_{4}$. The mole fractions of $\mathrm{CBr}_{4}$ are 0.1 and 0.25 . The refractive index of this system increases with increasing $\mathrm{CBr}_{4}$ content, so that $(\partial n / \partial c)_{p, T}$ is positive (cf. Fig. 2) and the refractive index increment with temperature $(\partial n / \partial T)_{p, C}$ is negative (cf. Fig. 2 ). The concentration part of the TDFRS signal increases exponentially (Fig. 5), which implies that the Soret coefficient is positive and the heavier component $\mathrm{CBr}_{4}$ migrates to 


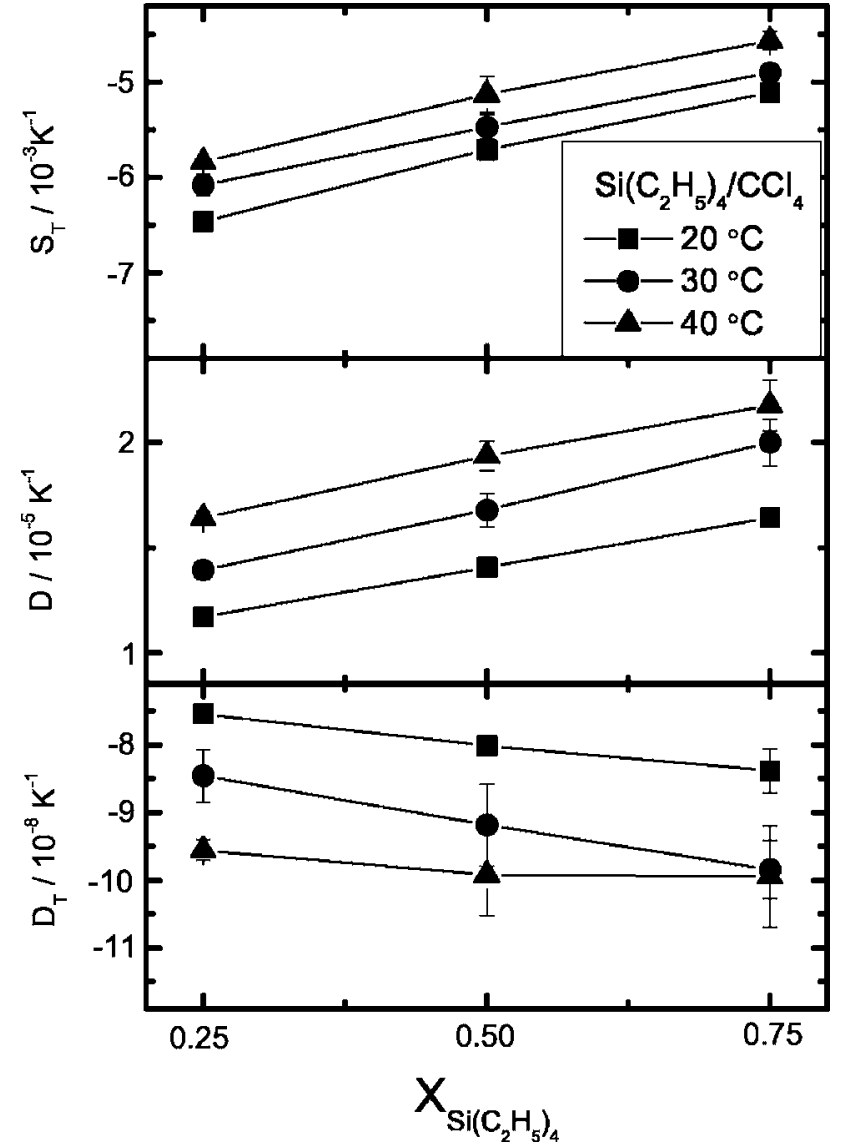

FIG. 7. Soret coefficient $S_{T}$, mutual diffusion coefficient $D$, and thermal diffusion coefficient $D_{T}$ of $\mathrm{Si}\left(\mathrm{C}_{2} \mathrm{H}_{5}\right)_{4}$ in $\mathrm{CCl}_{4}$ as a function of concentration.

the cold side. The diffusion coefficient increases with increasing temperature and decreases with increasing $\mathrm{CBr}_{4}$ content. This behavior can be explained by a lower viscosity at higher temperatures and higher $\mathrm{CBr}_{4}$ content.

In Figs. 7 and 8 the concentration dependence of $S_{T}, D$, and $D_{T}$ for tetraethysilane and di-tert-butylsilane is displayed. The Soret coefficient of both silanes is negative, which implies that the lighter silanes move to the warm side, while the heavier and denser $\mathrm{CCl}_{4}$ migrates to the cold. As in the case of $\mathrm{CBr}_{4}$, the rule of thumb that typically the heavier compound moves to the cold side is obeyed. The magnitude of the Soret coefficient for di-tert-butylsilane is roughly $10 \%$ larger than the one of tetraethylsilane. Both systems show a weak concentration dependence, which is typical for unpolar substances. $^{25,27}$ As expected, the diffusion coefficient increases with increasing temperature and with increasing amount of the less viscous silane. For all systems investigated, an increasing concentration of the heavier component leads to a decreasing diffusion coefficient.

\section{SIMULATIONS}

The reverse nonequilibrium molecular dynamics method has been applied to investigate the thermal diffusion of carbon tetrachloride in silanes. In the following, we only briefly summarize the force fields used for the liquid solvents and give some simulation details. A detailed description can be found elsewhere. ${ }^{28}$

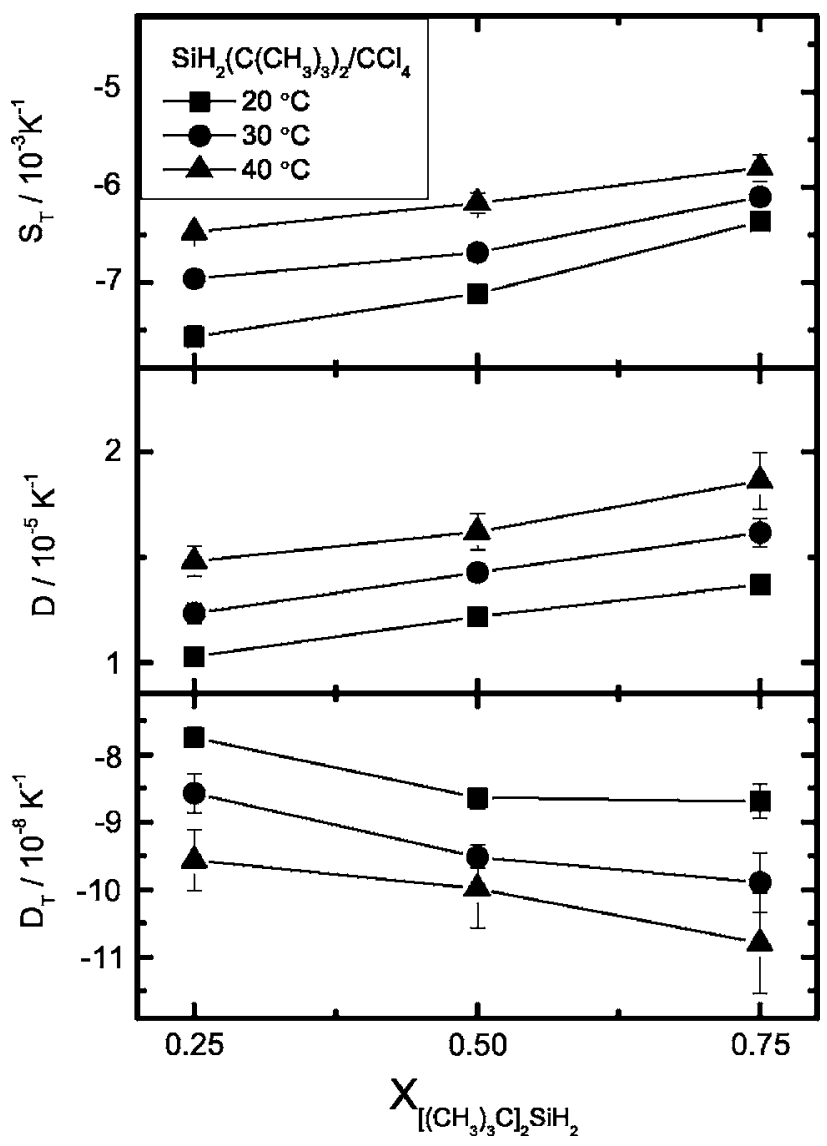

FIG. 8. Soret coefficient $S_{T}$, mutual diffusion coefficient $D$, and thermal diffusion coefficient $D_{T}$ of $\left[\left(\mathrm{CH}_{3}\right)_{3} \mathrm{C}\right]_{2} \mathrm{SiH}_{2}$ in $\mathrm{CCl}_{4}$ as a function of concentration.

\section{A. The force field for carbon tetrachloride}

In obtaining the force field for carbon tetrachloride we followed the work of Rey et al. ${ }^{29}$ They developed a consistent set of molecular models for MD simulations of a whole family of methylchloromethanes $\left(\left(\mathrm{CH}_{3}\right)_{4-n} \mathrm{CCl}_{n}\right)$, providing excellent accordance with thermodynamic properties (liquid density and heat of vaporization). It was found that nonnegligible electrostatic effects on the liquid structure exist only for the polar cases $(n=2,3)$. Therefore, we used the proposed force field for carbon tetrachloride without charges (see Table II).

\section{B. The force field for the silanes}

For tetraethylsilane we used a force field derived by Striolo et al. $^{30}$ Atoms in the same molecule interact with each other via short-range potentials that account for bond length, bond angle, and torsional potentials. It was shown that, by combining force fields independently developed to describe silsesquioxanes (POSS) and alkanes (Table II), it is possible to predict correctly the structure of isolated hybrid polyhedral oligomeric silsesquioxane monomers as well as that of crystals composed solely of POSS monomers. ${ }^{30}$

Test MD runs of a cubic periodic box of 200 tetraethylsilane molecules at $298 \mathrm{~K}$ and 1 atm yielded an unsatisfactory solvent density $\left(0.795 \mathrm{~kg} / \mathrm{m}^{3}\right)$ and heat of vaporization $(33.2 \mathrm{~kJ} / \mathrm{mol}$ ) (density $4.3 \%$ too high, heat of vaporization 
TABLE II. Lennard-Jones parameters for tetraethylsilane, di-tertbutylsilane, and carbon tetrachloride (* - Lennard-Jones parameters were adjusted to get experimental density and heat of vaporization).

\begin{tabular}{lll}
\hline \hline Atom type & $\sigma(\mathrm{nm})$ & $\varepsilon(\mathrm{kJ} / \mathrm{mol})$ \\
\hline $\mathrm{C}($ Ref. 29) & 0.3774 & 0.2277 \\
$\mathrm{Cl}($ Ref. 29) & 0.3467 & 1.0945 \\
$\mathrm{Si}^{*}$ & 0.5 & 0.42 \\
$\mathrm{SiH}_{2}{ }^{*}$ & 0.559 & 0.5 \\
$\mathrm{CH}_{2}$ Refs. 32 and 33 & 0.393 & 0.3808 \\
{$\left[\right.$ for Si($\left.\left(\mathrm{C}_{2} \mathrm{H}_{5}\right)_{4}\right]$} & & \\
$\mathrm{CH}_{3}$ Refs. 32 and 33 & 0.391 & 0.8647 \\
{$\left[\right.$ for $\left.\mathrm{Si}\left(\mathrm{C}_{2} \mathrm{H}_{5}\right)_{4}\right]$} & & \\
$\mathrm{C} \mathrm{Refs.} \mathrm{32} \mathrm{and} \mathrm{33}$ & 0.391 & 0.1413 \\
$\left(\right.$ for $\left.\left[\left(\mathrm{CH}_{3}\right)_{3} \mathrm{C}\right]_{2} \mathrm{SiH}_{2}\right)$ & & \\
$\mathrm{CH} \mathrm{H}_{3}$ Refs. 32 and 33 & 0.385 & 0.582 \\
$\left(\right.$ for $\left.\left[\left(\mathrm{CH}_{3}\right)_{3} \mathrm{C}\right]_{2} \mathrm{SiH}_{2}\right)$ & & \\
\hline \hline
\end{tabular}

$5.8 \mathrm{~kJ} / \mathrm{mol}$ too low). Experimental values at $298 \mathrm{~K}$ are presented in Table III. Another difficulty with this model is that it is only applicable to silanes with linear alkyl chain attached (the alkane backbone was modeled according to the TraPPE united-atom force field ${ }^{31}$ developed for the linear alkanes). Slightly different parameters were required for the $\mathrm{CH}_{3}$ groups of branched alkanes in order to reproduce the phase diagram. ${ }^{32,33}$ Therefore, we had to use a different route to describe di-tert-butylsilane, which has branched alkane structures attached.

In order to simulate both silanes consistently on the basis of the same force field parameters we derived new united atom force field parameters (Table II). For the alkane we applied the force field from Nath. ${ }^{32,33}$ In the case of tetraethylsilane, the Lennard-Jones parameters $\sigma$ and $\varepsilon$ of $\mathrm{Si}$, and for di-tert-butylsilane those of $\mathrm{SiH}_{2}$ have been adjusted. These parameters were increased to reproduce the experimental density and the heat of vaporization for the systems (Tables II and III).

\section{Computational details}

Two binary equimolar mixtures of carbon tetrachloride with tetraethylsilane and di-tert-butylsilane were simulated at $T=303 \mathrm{~K}$ and $P=1 \mathrm{~atm}$. The YASP package ${ }^{34}$ was used. The cell was elongated in the $z$ direction, which is the direction of the heat flow $\left(L_{x}=L_{y}=L_{z} / 3 \approx 4 \mathrm{~nm}\right)$. The cutoff length for nonbonded interactions was $1.2 \mathrm{~nm}$. The time step was $2 \mathrm{fs}$. All reverse noneqilibrium molecular-dynamics (RNEMD) simulations were performed at constant $N V T$ conditions (densities: 1047.2 and $1009.9 \mathrm{~kg} / \mathrm{m}^{3}$ for equimolar mixtures of tetraethylsilane and di-tert-butylsilane in carbon tetrachloride, respectively) with 960 molecules in the simulation box. The average temperature was kept constant by the thermostat of Berendsen et al., ${ }^{35}$ with the temperature coupling time being $T=1$ ps. Figures 9 and 10 show the concentration profiles for equimolar mixtures of tetraethylsilane and di-tertbutylsilane in $\mathrm{CCl}_{4}$ averaged over a simulation time of 51.9 and $74.12 \mathrm{~ns}$, respectively. The time development of the Soret coefficient for both mixtures is shown in Fig. 11 as cumulative average. For each silane, two values are displayed. One has been calculated from nine slabs of the downward branch and another from nine slabs in the upward branch. The hottest and coldest slabs have been excluded from the analysis. After $40 \mathrm{~ns}$, the $S_{T}$ value for tetraethylsilane $/ \mathrm{CCl}_{4}$ converges to a plateau value of -5 $\times 10^{-3} \mathrm{~K}^{-1}$. In the case of the system di-tert-butylsilane $/ \mathrm{CCl}_{4}$ the plateau $\left(-5.5 \times 10^{-3} \mathrm{~K}^{-1}\right)$ is reached later after $60 \mathrm{~ns}$. The average value $S_{T}=-5.5$ $\times 10^{-3} \mathrm{~K}^{-1}$ for di-tert-butylsilane in $\mathrm{CCl}_{4}$ is $10 \%$ smaller than the value obtained for tetraethylsilane in $\mathrm{CCl}_{4}$. The difference is not very pronounced, but this tendency is also confirmed in the experiment, although the magnitude of the experimental values is systematically $9 \%-18 \%$ larger.

\section{DISCUSSION}

\section{A. Comparison of the experimental and simulation results}

The following values for the Soret coefficient were determined by simulations at around $303 \mathrm{~K}$ for a binary equimolar mixtures of tetraethylsilane and di-tert-butylsilane in carbon tetrachloride: $-5 \pm 0.3 \times 10^{-3}$ and $-5.5 \pm 0.3$ $\times 10^{-3} \mathrm{~K}^{-1}$. The simulation results are larger than the experimental values and their magnitude is smaller by 9 and $18 \%$. These small systematic errors are probably due to the force field parameters, which were developed to reproduce the density and heat of vaporization of the pure components but not any transport coefficient. For gaseous systems it is known that the calculation of the Soret coefficient is much more sensitive to the correct choice of the interaction potential than other properties such as viscosity or diffusion coefficient. $^{36}$

Another reason might be the sensitivity of the Soret coefficient to the chosen mixing rule. Galliero et al. ${ }^{15}$ showed for methane/n-decane a strong dependence of $S_{T}$ on the cross-interaction parameters $k_{i, j}$ and $l_{i, j}$, which determine the interaction strengths $\varepsilon_{1,2}$,

TABLE III. Thermodynamic results obtained for pure tetraethylsilane, di-tert-butylsilane, and carbon tetrachloride compared with the corresponding experimental results at $298 \mathrm{~K}$.

\begin{tabular}{|c|c|c|c|c|}
\hline Solvent & $\rho_{\text {exp }}\left(\mathrm{kg} / \mathrm{m}^{3}\right)$ & $\rho_{\text {sim }}\left(\mathrm{kg} / \mathrm{m}^{3}\right)$ & $\Delta H_{\text {vap }}^{\exp }(\mathrm{kJ} / \mathrm{mol})$ & $\Delta H_{\text {vap }}^{\text {sim }}(\mathrm{kJ} / \mathrm{mol})$ \\
\hline \multirow[t]{2}{*}{$\mathrm{Si}\left(\mathrm{C}_{2} \mathrm{H}_{5}\right)_{4}$} & 0.761 & 0.773 & 39 & 37.9 \\
\hline & Ref. 17 & & Ref. 19 & \\
\hline \multirow{2}{*}[(\mathrm{CH}_{3})_{3}\mathrm{C}]{$_{2} \mathrm{SiH}_{2}$} & 0.729 & 0.735 & $\cdots$ & 38.1 \\
\hline & Ref. 17 & & & \\
\hline \multirow[t]{2}{*}{$\mathrm{CCl}_{4}$} & 1.594 & 1.588 & 31.5 & 30.06 \\
\hline & Ref. 17 & & Ref. 20 & \\
\hline
\end{tabular}




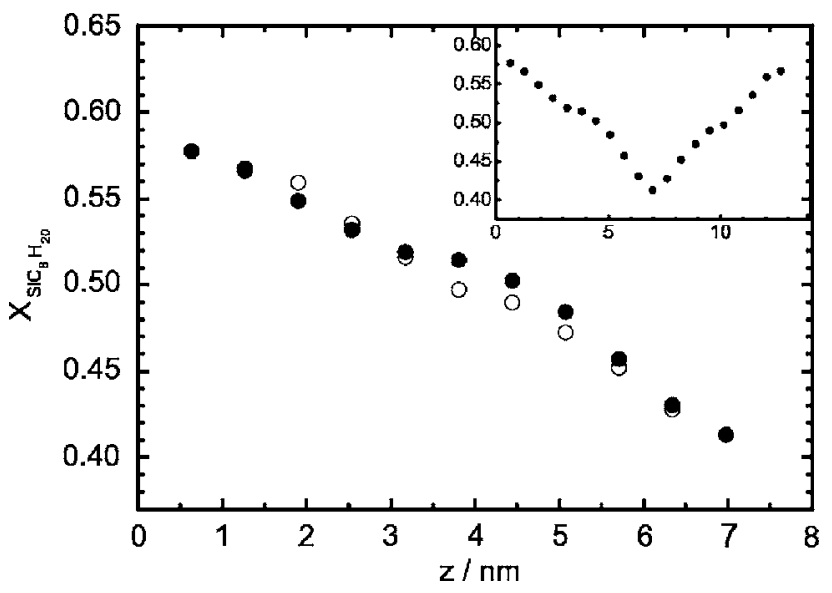

FIG. 9. Concentration profiles for a equimolar mixture of tetraethylsilane/ $\mathrm{CCl}_{4}$ averaged over a simulation time of $52 \mathrm{~ns}$. The inset shows the complete concentration profile. The open symbols represent the upward branch of the full profile, which has been flipped over at the coldest slab.

$$
\begin{aligned}
& \varepsilon_{1,2}=\left(1-k_{i, j}\right)\left(\varepsilon_{1} \varepsilon_{2}\right)^{0.5}, \\
& \sigma_{1,2}=\left(1-l_{i, j}\right) \frac{1}{2}\left(\sigma_{1}+\sigma_{2}\right)
\end{aligned}
$$

with $\varepsilon_{1,2}, \varepsilon_{1}, \varepsilon_{2}$ the interaction strength parameters and $\sigma_{1,2}, \sigma_{1}, \sigma_{2}$ the diameters.

In our case the classic Lorenz-Berthelot mixing rule $\left(k_{i, j}=0 ; l_{i, j}=0\right)$ was used, which had been applied in the simulation of benzene/cyclohexane ${ }^{28}$ mixtures. Unfortunately, a validation of the cross-interaction parameters was impossible, because to our best knowledge there are no literature data available for the investigated mixtures. In view of other MD simulations of the Soret coefficient of fluid mixtures, some of which use much more sophisticated force fields, ${ }^{28}$ the agreement with experiment must be viewed as very satisfactory.

\section{B. Contributions to the Soret coefficient}

Galliero et al. $^{15}$ investigated the thermodiffusion behavior of equimolar mixtures of "super methane" in methane.

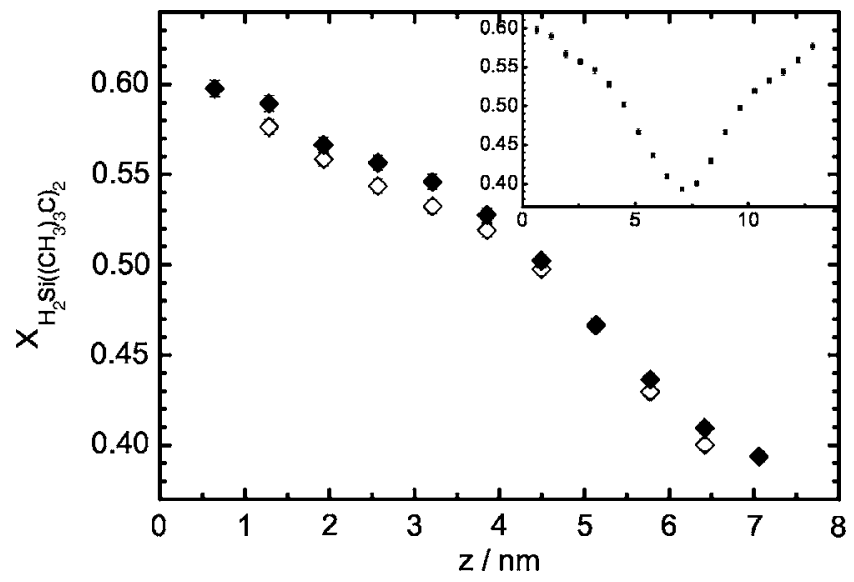

FIG. 10. Concentration profiles for a equimolar mixture of di-tert-butylsilane/ $\mathrm{CCl}_{4}$ averaged over a simulation time of $55 \mathrm{~ns}$. The inset shows the complete concentration profile. The open symbols represent the upward branch of the full profile, which has been flipped over at the coldest slab.

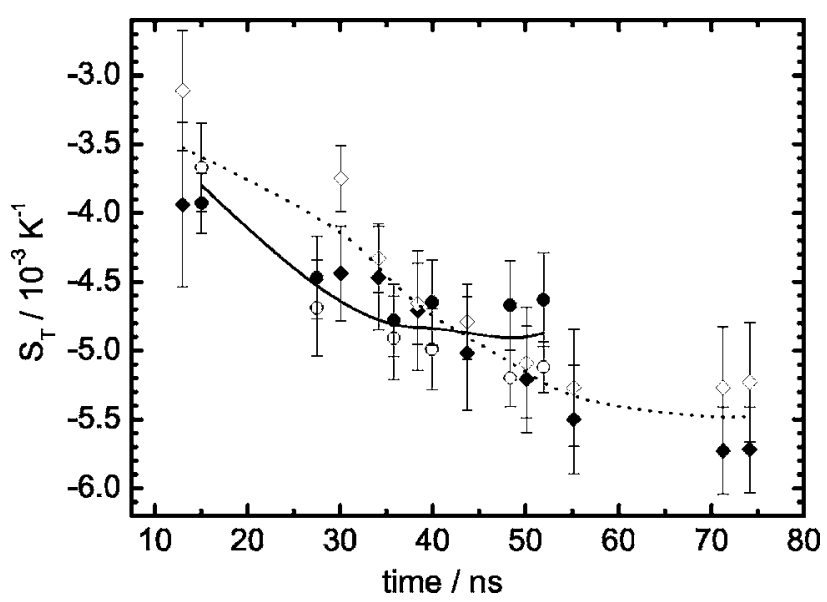

FIG. 11. Soret coefficients of the tetraethylsilane (circles) and di-tertbutylsilane (diamonds) in carbon tetrachloride as a function of simulation time. One set of values has been calculated from the downward (solid symbols) branch and the other values have been calculated from the upward branch (open symbol). The lines are guides to the eye for tetraethylsilane (solid line) and di-tert-butylsilane (dotted line).

The parameters mass, $m$, diameter, $\sigma$ and depth of the interaction potential, $\varepsilon$ of super methane were different from those of methane. As already done earlier by Reith et al., ${ }^{12}$ they varied systematically the ratio of one of the parameters (e.g., $m_{1} / m_{2}$ ) while keeping the other two parameters fixed and equal. By this procedure they obtained three additive contributions $\alpha_{T}^{m}, \alpha_{T}^{\sigma}$, and $\alpha_{T}^{\varepsilon}$ of the total thermal diffusion ratio $\alpha_{T}=S_{T} T$ stemming from the mass, diameter and interaction strength, respectively.

$$
\alpha_{T}=\alpha_{T}^{m}+\alpha_{T}^{\sigma}+\alpha_{T}^{\varepsilon} .
$$

This empirical formula reproduces the simulation results quite well if the ratios do not deviate too much from 1.

We applied Eq. (8) to the investigated mixtures of tetraethylsilane, di-tert-butylsilane, and carbon tetrabromide in carbon tetrachloride (component 2). The ratios of the diameter and the depths of the interaction potential were estimated using the experimental molar volumes $V_{\text {mol }}$ (at room temperature) and enthalpies of vaporization $H_{\text {vap }}$ (at boiling point) for the different components, ${ }^{37}$

$$
\begin{aligned}
& \frac{\sigma_{1}}{\sigma_{2}}=\left(\frac{V_{\mathrm{mol}, 1}}{V_{\mathrm{mol}, 2}}\right)^{1 / 3}, \\
& \frac{\varepsilon_{1}}{\varepsilon_{2}}=\left(\frac{\Delta H_{\mathrm{vap}, 1}}{\Delta H_{\mathrm{vap}, 2}}\right) .
\end{aligned}
$$

The results are summarized in Table IV. The mass contribution for $\mathrm{CBr}_{4} / \mathrm{CCl}_{4}$ mixture is positive while for tetraethylsilane $/ \mathrm{CCl}_{4}$ and di-tert-butylsilane $/ \mathrm{CCl}_{4}$ it is negative. This implies that the component with the higher molar mass moves to the cold side. The mass contributions for both tetraethylsilane $/ \mathrm{CCl}_{4}$ and di-tert-butylsilane $/ \mathrm{CCl}_{4}$ are the same. At the same time the difference in size and, even more pronounced, the difference in the interaction potential leads to a stronger drive of di-tert-butylsilane to the hot side. The values obtained from Eqs. (8)-(10) reproduce the correct direction of thermodiffusion motion for all three mixtures, but they are one order of magnitude too small. 
TABLE IV. The values of the Soret coefficient calculated using Eq. (8) at $303 \mathrm{~K}$. The deviations from the experimental values (equimolar mixtures) are shown in brackets.

\begin{tabular}{|c|c|c|c|c|c|}
\hline Solute & $S_{T}^{m}\left(10^{-3} \mathrm{~K}^{-1}\right)$ & $S_{T}^{\sigma}\left(10^{-3} \mathrm{~K}^{-1}\right)$ & $S_{T}^{\varepsilon}\left(10^{-3} \mathrm{~K}^{-1}\right)$ & $\begin{array}{l}S_{T} \text { from Eq. (8) } \\
\left(10^{-3} \mathrm{~K}^{-1}\right)\end{array}$ & Measured $S_{T}\left(10^{-3} \mathrm{~K}^{-1}\right)$ \\
\hline $\mathrm{Si}\left(\mathrm{C}_{2} \mathrm{H}_{5}\right)_{4}$ & -0.15 & -0.33 & 0.382 & $\begin{array}{l}-0.11 \\
(98 \%)\end{array}$ & -5.5 \\
\hline$\left[\left(\mathrm{CH}_{3}\right)_{3} \mathrm{C}\right]_{2} \mathrm{SiH}_{2}$ & -0.15 & -0.36 & 0.26 & $\begin{array}{l}-0.25 \\
(96 \%)\end{array}$ & -6.7 \\
\hline $\mathrm{CBr}_{4}$ & 1.77 & -0.07 & 0.57 & 2.26 & $\sim 12$ (extrapolated from Fig. 6 ) \\
\hline
\end{tabular}

The values of the Soret coefficient can also be compared with a parameter of the cohesive energy density, ${ }^{11-14}$ which is also referred to as the Hildebrand solubility parameter. As it is expected in our case the component with the larger Hildebrand parameter (cf. Table I) carbon tetrabromide (mixed with carbon tetrachloride) and carbon tetrachloride (mixed with one of the silanes) accumulates in the cold region.

\section{Discussion of the effect of the moment of inertia}

The importance of the moments of inertia in the thermal diffusion behavior of the benzene/cyclohexane mixture was reported by Debuschewitz et al. ${ }^{7}$ They found that the component with the larger $I_{z}$ (the main moment of inertia perpendicular to the plane of the molecule) moves to the cold side. The physical reason for this phenomenon in liquids is not clear. In an old work by Schirdewahn et al. ${ }^{38}$ about gaseous mixtures, the moment of inertia part is vaguely related to the rotational diffusion contribution to the thermal diffusion. At the same time, it is obvious that the values of the main moments of inertia for a given molecule characterize its size (or van der Waals volume) and the mass distribution, while all three possible ratios $\left(I_{x} / I_{y}, I_{x} / I_{z}, I_{y} / I_{z}\right)$ characterize the shape. All these factors are responsible for the diffusion (as well as the thermodiffusion) behavior. From this point, one can assume that the value of $I_{z}$ in the case of benzene/ cyclohexane mixtures is responsible not only for the size but also for the mass distribution in the molecule, but not for the shape. They assumed that both molecules have a disklike structure, which is not changed by isotopic substitution. In our case carbon tetrachloride, carbon tetrabromide, and tetraethylsilane have spherical structures as far as all diagonal elements of their tensors of the moment of inertia are the same (cf. Table I). The lower value of $I_{x}$ in comparison to the values of $I_{y}$ and $I_{z}$ for di-tert-butylsilane describes the ellipsoidal shape of the molecule. In order to take into account the shape effect, a more complicated expression for $S_{T}^{I}$, involving $I_{x}, I_{y}$, and $I_{z}$ for binary mixtures of equivolume components is required. However, the differences in $S_{T}$ between tetraethylsilane and di-tert-butylsilane are too small to be unambiguously attributed to differences in the moment of inertia. Other effects, such as the anisotropic friction, could also explain an effect of this magnitude.

\section{CONCLUSION}

We investigated the thermal diffusion behavior of three simple mixtures consisting of spherical molecules experimentally by the so-called TDFRS method. Additionally, we applied a molecular exchange version of the reverse nonequilibrium molecular dynamics algorithm to determine the Soret coefficients for two of the experimentally investigated mixtures.

Contrary to more complex fluids, the observed thermophoretic motion for those three unpolar mixtures follows the common rules, which state that the component with the larger molar mass and the larger Hildebrandt parameter accumulates in the cold region.

We found a fairly good agreement between the simulated and experimentally determined Soret coefficients for the systems tetraethylsilane and di-tert-butylsilane in carbon tetrachloride. Although the magnitude of the simulated $S_{T}$ values is systematically smaller by $9-18 \%$ than in experiment, both methods found that di-tert-butylsilane accumulates slightly stronger in the warm region than the more symmetric tetraethylsilane. Both silane compounds investigated do have the same molar mass, so we tried to reveal the moment of inertia contribution to the thermophoretic motion. Here, it turned out that the analysis is not so simple because two of the three moments of inertia are changed simultaneously. This requires an expression which connects the Soret coefficient with more than one moment of inertia. In order to obtain such a relation more systematic experiments and simulations need to be performed in the future. In addition, the difference between the two silanes is very small.

\section{ACKNOWLEDGMENTS}

The authors would like to thank E. Rossinsky for helpful discussion. The authors thank the Deutsche Forschungsgemeinschaft and the John von Neumann Institute for Computing at the Forschungszentrum Juelich, which provided the computer time for this study.

\footnotetext{
${ }^{1}$ M. E. Schimpf and J. C. Giddings, Macromolecules 20, 1561 (1987).

${ }^{2}$ K. Clusius and G. Dickel, Naturwissenschaften 27, 148 (1939).

${ }^{3}$ H. C. Helgeson, Pure Appl. Chem. 57, 31 (1885).

${ }^{4}$ P. Costeseque, D. Fargue, and P. Jamet, in Thermal Nonequilibrium Phenomena in Fluid Mixtures, Lecture Notes in Physics, edited by W. Köhler and S. Wiegand (Springer, Berlin, 2000), pp. 389-427.

${ }^{5}$ D. E. Rosner, R. S. Israel, and B. La Mantia, Combust. Flame 123, 547 (2000).

${ }^{6}$ S. Wiegand, J. Phys.: Condens. Matter 16, R357 (2004).

${ }^{7}$ C. Debuschewitz and W. Köhler, Phys. Rev. Lett. 87, 055901 (2001).
} 
${ }^{8}$ G. Wittko and W. Köhler, J. Chem. Phys. 123, 014506 (2005).

${ }^{9}$ P. Polyakov, J. Luettmer-Strathmann, and S. Wiegand, J. Phys. Chem. B 110, 26215 (2006).

${ }^{10}$ P.-A. Artola and B. Rousseau, Phys. Rev. Lett. 98, 125901 (2007).

${ }^{11}$ J. Demichowicz-Pigoniowa, M. Mitchell, and H. Tyrrell, J. Chem. Soc. A 2, 307 (1971).

${ }^{12}$ D. Reith and F. Müller-Plathe, J. Chem. Phys. 112, 2436 (2000).

${ }^{13}$ E. P. C. Mes, W. T. Kok, and R. Tijssen, Int. J. Polym. Anal. Charact. 8, 133 (2003).

${ }^{14}$ R. Kita, P. Polyakov, and S. Wiegand, Macromolecules 40, 1638 (2007).

${ }^{15}$ G. Galliéro, B. Duguay, J. P. Caltagirone, and F. Montel, Fluid Phase Equil. 208, 171 (2003).

${ }^{16}$ Chem3D, Ver.10, CambridgeSoft, Cambridge, MA (2006).

${ }^{17}$ The molar mass, the density and the boiling point information were taken datasheet for the chemical provided by the company Aldrich. See http:// www.sigmaaldrich.com

${ }^{18}$ F. Trouton, Philos. Mag. 18, 54 (1884).

${ }^{19}$ J. Chickos and W. Acree, J. Phys. Chem. Ref. Data 32, 519 (2003).

${ }^{20}$ C. Yaws, Chemical Properties Handbook (McGraw Hill, New York, 1999).

${ }^{21}$ We found the density for carbon tetrabromide at https://www.wpi.edu/ Academics/Depts/Chemistry/Courses/General/flotation.html and http:// 1rc-srvr.mps.ohio-state.edu/under/chemed/qbank/7/7-3.que

${ }^{22}$ J. Edward, J. Chem. Educ. 47, 261 (1970).
${ }^{23}$ C. Yokoyama, T. Takagi, and S. Takahashi, Int. J. Thermophys. 11, 477 (1990).

${ }^{24}$ A. Becker, W. Köhler, and B. Müller, Ber. Bunsenges. Phys. Chem 99, 600 (1995).

${ }^{25}$ A. Perronace, C. Leppla, F. Leroy, B. Rousseau, and S. Wiegand, J. Chem. Phys. 116, 3718 (2002).

${ }^{26}$ R. L. Saxton, E. L. Dougherty, and H. G. Drickamer, J. Chem. Phys. 22, 1166 (1954).

${ }^{27}$ W. Köhler and B. Müller, J. Chem. Phys. 103, 4367 (1995).

${ }^{28}$ M. Zhang and F. Müller-Plathe, J. Chem. Phys. 123, 124502 (2005).

${ }^{29}$ R. Rey, L. Pardo, E. Llanta, K. Ando, D. Lopez, J. Tamarit, and M. Barrio, J. Chem. Phys. 112, 7505 (2000).

${ }^{30}$ A. Striolo, C. McCabe, and P. Cummings, J. Chem. Phys. 125, 104904 (2006).

${ }^{31}$ G. Martin and J. Siepmann, J. Phys. Chem. B 102, 2569 (1998).

${ }^{32}$ S. Nath and R. Khare, J. Chem. Phys. 115, 10837 (2001).

${ }^{33}$ J. Wescott, P. Kung, and S. Nath, Fluid Phase Equilib. 208, 123 (2003).

${ }^{34}$ F. Müller-Plathe, Comput. Phys. Commun. 78, 77 (1993).

${ }^{35}$ H. Berendsen, J. Chem. Phys. 81, 3684 (1984).

${ }^{36}$ L. Waldmann, in Handbuch der Physik, edited by S. Flüegge (Springer, Berlin, 1958), Vol. 12, pp. 295-514.

${ }^{37}$ D. Reith, Ph.D. thesis, Universität Mainz und Max-Planck-Institut für Polymerforschung (1998).

${ }^{38}$ J. Schirdewahn, A. Klemm, and L. Waldmann, Z. Naturforsch. A 16A, 133 (1961). 